TRANSACTIONS OF THE

AMERICAN MATHEMATICAL SOCIETY

Volume 353, Number 9, Pages 3617-3627

S 0002-9947(01)02745-3

Article electronically published on April 18, 2001

\title{
MAXIMAL DEGREE SUBSHEAVES OF TORSION FREE SHEAVES ON SINGULAR PROJECTIVE CURVES
}

\author{
E. BALLICO
}

\begin{abstract}
Fix integers $r, k, g$ with $r>k>0$ and $g \geq 2$. Let $X$ be an integral projective curve with $g:=p_{a}(X)$ and $E$ a rank $r$ torsion free sheaf on $X$ which is a flat limit of a family of locally free sheaves on $X$. Here we prove the existence of a rank $k$ subsheaf $A$ of $E$ such that $r(\operatorname{deg}(A)) \geq$ $k(\operatorname{deg}(E))-k(r-k) g$. We show that for every $g \geq 9$ there is an integral projective curve $X, X$ not Gorenstein, and a rank 2 torsion free sheaf $E$ on $X$ with no rank 1 subsheaf $A$ with $2(\operatorname{deg}(A)) \geq \operatorname{deg}(E)-g$. We show the existence of torsion free sheaves on non-Gorenstein projective curves with other pathological properties.
\end{abstract}

\section{INTRODUCTION}

A classical theorem of C. Segre and M. Nagata gives an upper bound (i.e., $g$ ) for the minimal self-intersection of a section of a ruled surface over a projective curve $X$ of genus $g$. If the ruled surface is isomorphic to $\mathbf{P}(E)$ with $E$ rank two vector bundle on $X$ and $L$ is a rank 1 subbundle of $E$ with $\operatorname{deg}(L)$ maximal, this theorem is equivalent to the inequality $\operatorname{deg}(E)-2(\operatorname{deg}(L)) \leq g$. This result was extended by S. Mukai and F. Sakai to the case of vector bundles on $X$ ([15]). In this paper we want to study the corresponding problem for a singular curve. On a singular curve it is natural to consider torsion free sheaves and not only locally free sheaves. However, in general we give an affirmative answer to the corresponding problem only for a torsion free sheaf which is a flat limit of a flat family of locally free sheaves (see Theorem 0.1). This is not a restrictive assumption if and only if $X$ has only planar singularities ([17]).

Let $X$ be a reduced and irreducible projective curve of genus $g$ defined over an algebraically closed field $\mathbf{K}$ with arbitrary characteristic. For any coherent sheaf $F$ on $X$ whose generic rank is $r$ set $\operatorname{deg}(F):=\chi(F)+r(g-1)=\chi(F)-r \chi\left(O_{X}\right)$ and, if $r>0, \mu(F):=\operatorname{deg}(F) / r$. Fix integers $r, k$ with $r>k>0$. Let $E$ be a rank $r$ torsion free sheaf on $X$ and $A$ a rank $k$ subsheaf of $E$ such that $\operatorname{deg}(A)$ is the maximal degree of a rank $k$ subsheaf of $E$. Set $s_{k}(E):=k(\operatorname{deg}(E))-r(\operatorname{deg}(A))$ and call the integer $s_{k}(E), 1 \leq k \leq r-1$, a Lange invariant of $E$. The name is due to the nice use of these invariants in the case of a smooth curve made in 11]. Notice that $A$ is saturated in $E$ (i.e. $E / A$ has no torsion) by the maximality of $\operatorname{deg}(A)$ and that $s_{k}(E)=k(r-k)(\mu(E / A)-\mu(A))$. Notice that $E$ is stable (resp. semistable) if and only if $s_{k}(E)>0$ (resp. $s_{k}(E) \geq 0$ ) for every integer $k$ with $1 \leq k \leq r-1$.

In the first section of this paper we prove the following result.

Received by the editors September 25, 1998.

2000 Mathematics Subject Classification. Primary 14H20, 14H60.

(C)2001 American Mathematical Society 
Theorem 0.1. Let $X$ be an integral projective curve of arithmetic genus $g \geq 2$. Fix integers $r, k$ with $r>k>0$ and let $E$ be a rank $r$ torsion free sheaf on $X$ which is the flat limit of a family of locally free sheaves. Then $s_{k}(E) \leq \operatorname{gk}(r-k)$.

The main aim of this paper is to show that torsion free sheaves on non-Gorenstein projective curves may have several pathological properties (see section 2). One of our examples gives the following result.

Theorem 0.2. For every integer $g \geq 9$ there exists an integral projective curve $X$ with $g=p_{a}(X)$ with a rank 2 torsion free sheaf $E$ on $X$ such that $s_{1}(E)>g$.

For another pathological property, see Theorem 2.6

The author wants to thank V. Barucci for several e-mail conversations. The author was partially supported by MURST and GNSAGA of CNR (Italy).

\section{ProOF OF 0.1}

Fix $Q \in X_{\text {reg }}$ and a rank $r$ torsion free sheaf $F$ on $X$. For any $Q \in X, \mathbf{K}_{Q}$ will denote the skyscraper sheaf on $X$ with $Q$ as support and $h^{0}\left(X, \mathbf{K}_{Q}\right)=1$. Let $F \mid\{Q\}$ be the fiber of $F$ over $Q$. Since $F$ has rank $r, F \mid\{Q\}$ is an $r$-dimensional vector space over $\mathbf{K}$. Fix a linear surjective map $f(Q): F \mid\{Q\} \rightarrow \mathbf{K}$ or, equivalently, a surjection $f: F \rightarrow \mathbf{K}_{Q}$. $\operatorname{Ker}(f)$ is a torsion free sheaf on $X$ which has the same formal completion as $F$ at each point of $\operatorname{Sing}(X)$. Following [14] and [1] we will say that $\operatorname{Ker}(f)$ is obtained from $F$ making a negative elementary transformation supported by $Q$ and that $F$ is obtained from $\operatorname{Ker}(f)$ making a positive elementary transformation supported by $Q$. Indeed, since $Q \in X_{\text {reg, the local to }}$ global spectral sequence for the Ext-functors shows that $F$ is uniquely determined by the choice of $\operatorname{Ker}(f)$ and of a linear surjective map from $(\operatorname{Ker}(f) \mid\{Q\})^{*}$ to $\mathbf{K}$. More generally, we may take an integer $x$ with $0<x \leq r$ and a linear surjective map $h(Q): F \mid\{Q\} \rightarrow \mathbf{K}^{\oplus x}$, i.e. a surjection $h: F \rightarrow \mathbf{K}_{Q}^{\oplus x}$. $\operatorname{Ker}(h)$ is a torsion free sheaf with $\operatorname{deg}(\operatorname{Ker}(h))=\operatorname{deg}(F)-x$. We will say that $\operatorname{Ker}(h)$ is obtained by a negative elementary transformation associated to the $(r-k)$-dimensional subspace $\operatorname{Ker}(h) \mid\{Q\}$ of $F \mid\{Q\}$. The sheaf $\operatorname{Ker}(h)$ may be obtained from $F$ making $x$ suitable negative elementary transformations supported by $Q$.

Remark 1.1. If $L \in \operatorname{Pic}(X)$ we have $s_{k}(E)=s_{k}(E \otimes L)$ for every torsion free sheaf $E$ because the map $H \rightarrow H \otimes L$ between rank $k$ subsheaves of $E$ and rank $k$ subsheaves of $E \otimes L$ has as inverse the map $T \rightarrow T \otimes L^{*}$ and $\operatorname{deg}(E \otimes L)=\operatorname{det}(E)+r(\operatorname{deg}(L))$, $\operatorname{deg}(H \otimes L)=\operatorname{deg}(H)+k(\operatorname{deg}(L))$. In particular we have $s_{k}(E)=s_{k}(E(-Q))$ for every $Q \in X_{\text {reg }}$.

The proof of the following key lemma was inspired by the proof of [12], Prop. 4.5 .

Lemma 1.2. Fix integers $r, k$ with $r>k>0$, an integral quasi-projective variety $T$ with $\operatorname{dim}(T)>0$, an integral projective curve $X, Q \in X_{\mathrm{reg}}$ and a torsion free sheaf $E$ on $X$ with $\operatorname{rank}(E)=r$. Assume the existence of a flat family of rank $k$ saturated subsheaves $\left\{F_{t}\right\}_{t \in T}$ of $E$ such that for every subsheaf $F$ of $E$ we have $F=F_{t}$ (as subsheaves of $E$ ) for at most finitely many $t \in T$ and such that all the fibers $\left\{F_{t} \mid\{Q\}\right\}_{t \in T}$ over the point $Q$ of the sheaves $F_{t}$ are the same $k$-dimensional linear subspace of the vector space $E \mid\{Q\}$. Then there is a rank $k$ subsheaf $G$ of $E$ with $\operatorname{deg}(G)>\operatorname{deg}\left(F_{t}\right)$ for any $t$. 
Proof. In order to obtain a contradiction we may assume that any $F_{t}$ computes the maximal degree of a rank $k$ subsheaf of $E$, i.e. that $s_{k}(E)=k(\operatorname{deg}(E))-r\left(\operatorname{deg}\left(F_{t}\right)\right)$ for all $t$. Set $U:=F_{t} \mid\{Q\}$ (seen as a $k$-dimensional linear subspace of the vector space $E \mid\{Q\}$ ) and let $V$ be any complementary codimension $k$ linear subspace of $U$ in $E \mid\{Q\}$. Call $V^{\prime \prime}$ the skyscraper sheaf on $X$ supported by $Q$ and with $(E \mid\{Q\} / U)$ as fiber. Let $E^{\prime}$ be the subsheaf of $E$ obtained from $E$ making the negative elementary transformation corresponding to the natural surjection $E \rightarrow$ $W^{\prime \prime}$. Since $U \cap V=\{0\}, V$ induces a unique linear subspace $V^{\prime}$ of $E^{\prime} \mid\{Q\}$ with $\operatorname{dim}\left(V^{\prime}\right)=r-k$. Call $E^{\prime \prime}$ the subsheaf of $E^{\prime}$ obtained from $E^{\prime}$ making the negative elementary transformation corresponding to $V^{\prime}$. Since $U+V=E \mid\{Q\}$ we have $E^{\prime \prime} \cong E(-Q)$. Every sheaf $F_{t}$ induces a subsheaf $F_{t}^{\prime}$ of $F_{t}$ with $F_{t}^{\prime} \cong F_{t}(-Q)$, but in general the fibers $\left\{F_{t}^{\prime} \mid\{Q\}\right\}_{t \in T}$ will not be constant. Every sheaf $F_{t}^{\prime}$ induces a subsheaf $F_{t}^{\prime \prime}$ of $E^{\prime \prime}$ and if the fibers $F_{t}^{\prime \prime} \mid\{Q\}$ at $Q$ of these subsheaves are not constant, then in general we will not obtain a flat family of subsheaves of $E^{\prime \prime}$ and the integer $\operatorname{deg}\left(F_{t}^{\prime \prime}\right)$ may depend on $t$. Assume that these fibers are not constant. Then we have at least one $t \in T$ with $F_{t}^{\prime} \mid\{Q\} \cap V^{\prime} \neq\{0\}$. Fix any such $t$. We have $\operatorname{deg}\left(F_{t}^{\prime \prime}\right)<\operatorname{deg}\left(F_{t}^{\prime}\right)=\operatorname{deg}\left(F_{t}\right)-k$ and hence

$$
\begin{aligned}
s_{k}(E(-Q)) & \leq k(\operatorname{deg}(E(-Q)))-r\left(\operatorname{deg}\left(F_{t}^{\prime \prime}\right)\right) \\
& <k(\operatorname{deg}(E(-Q)))-r(\operatorname{deg}(F(-Q)))=s_{k}(E),
\end{aligned}
$$

a contradiction. Now we assume that the family of fibers $\left\{F_{t}^{\prime} \mid\{Q\}\right\}_{t \in T}$ is constant. This implies that the restriction of the family $\left\{F_{t}\right\}_{t \in T}$ to the first infinitesimal neighborhood of $Q$ in $X$ is constant. We repeat the construction finitely many times until the corresponding family of fibers over $Q$ is not constant. Since the family of subsheaves $\left\{F_{t}\right\}_{t \in T}$ of $E$ is not constant and a saturated subsheaf, $F$, of $E$ is uniquely determined by the completion of the inclusion of $F$ in $E$ at $Q$, it is obvious that after finitely many steps the family of the fibers over $Q$ of the corresponding subsheaves is not constant. At that time using the first part of the proof we will obtain a rank $r$ sheaf $E_{1}$ and a family of rank $k$ subsheaves $\left\{F_{1 t}\right\}_{t \in T}$ of $E_{1}$ which does not compute $s_{k}\left(E_{1}\right)$, i.e. such that there is a rank $k$ saturated subsheaf $G_{1}$ of $E_{1}$ with $\operatorname{deg}\left(G_{1}\right)>\operatorname{deg}\left(F_{1 t}\right)$. $E_{1}$ is obtained from $E$ making a sequence of negative elementary transformations. Making in the reverse order the inverse positive elementary transformations and taking at each step the saturation of $G_{1}$ we obtain a rank $k$ subsheaf $G$ of $E$ with $\operatorname{deg}(G)>\operatorname{deg}\left(F_{t}\right)$.

Since the Grassmannian $G(k, r)$ has dimension $k(r-k)$, Lemma[1.2 gives at once the following result.

Corollary 1.3. Let $X$ be an integral projective curve and $E$ a rank $r$ torsion free

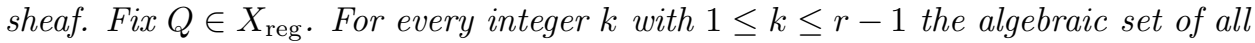
rank $k$ subsheaves of $E$ with maximal degree has dimension at most $k(r-k)$ and for any such family $\left\{F_{t}\right\}_{t \in T}$ the map from $T$ into the Grassmannian $G(n-k, E \mid\{Q\})$ of $k$-dimensional subspaces of $E \mid\{Q\}$ associating $F_{t} \mid\{Q\}$ to $t$ is quasi-finite.

Lemma 1.4. Fix integers $r, k, u, g$ with $r>k>0, u>0, g \geq 1$, an integral quasi-projective variety $T$ with $\operatorname{dim}(T)>u k(r-k)$, an integral projective curve $X$ with $p_{a}(X)=g$ and a torsion free sheaf $E$ on $X$ with $\operatorname{rank}(E)=r$. Assume the existence of a flat family of rank $k$ saturated subsheaves $\left\{F_{t}\right\}_{t \in T}$ of $E$ such that for every rank $k$ subsheaf $F$ of $E$ we have $F=F_{t}$ (as subsheaves of $E$ ) for at most finitely many $t \in T$. Then $s_{k}(E) \leq k(\operatorname{deg}(E))-r\left(\operatorname{deg}\left(F_{t}\right)\right)-2 u$. 
Proof. Lemma 1.2 and Corollary 1.3 cover the case $u=1$. Hence we may assume $u \geq 2$. Fix $Q_{i} \in X_{\text {reg }}, 1 \leq i \leq u$. Since $\operatorname{dim}(G(k, r))=k(r-k)$, there is at least a $k$-dimensional subspace, $U_{i}$, of $E \mid\left\{Q_{i}\right\}$ such that there is a subvariety $T^{\prime}$ of $T$ with $\operatorname{dim}\left(T^{\prime}\right) \geq \operatorname{dim}(T)-u k(r-k)>0$ and such that $F_{t} \mid\left\{Q_{i}\right\}=U_{i}$ for every $t \in T^{\prime}$ and every integer $i$ with $1 \leq i \leq u$. Let $W_{i}^{\prime \prime}$ be the skyscraper sheaf on $X$ supported by $Q$ and with $E \mid\left\{Q_{i}\right\} / U_{i}$ as fiber over $Q$. Let $V_{i}$ be any complementary codimension $k$ linear subspace of $U_{i}$ in $E \mid\{Q\}$. Let $E^{\prime}$ be the subsheaf of $E$ obtained from $E$ making for all integers $i$ with $1 \leq i \leq u$ the negative elementary transformation corresponding to the natural surjection $E \rightarrow W_{i}^{\prime \prime}$. Hence $\operatorname{deg}\left(E^{\prime}\right)=\operatorname{deg}(E)-u k$. Since $U_{i} \cap V_{i}=\{0\}$ for every $i, V_{i}$ induces a unique linear subspace $V_{i}^{\prime}$ of $E^{\prime} \mid\left\{Q_{i}\right\}$ with $\operatorname{dim}\left(V^{\prime}\right)=r-k$. Call $E^{\prime \prime}$ the subsheaf of $E^{\prime}$ obtained from $E^{\prime}$ making for all integers $i$ with $1 \leq i \leq u$ the negative elementary transformation corresponding to $V_{i}^{\prime}$. Since $U_{i}+V_{i}=E \mid\left\{Q_{i}\right\}$ for every $i$, we have $E^{\prime \prime} \cong E\left(-\sum_{1 \leq i \leq u} Q_{i}\right)$. Every sheaf $F_{t}, t \in T^{\prime}$, induces a subsheaf $F_{t}^{\prime}$ of $F_{t}$ with $F_{t}^{\prime} \cong F_{t}\left(-\sum_{1 \leq i \leq u} Q_{i}\right)$. Every sheaf $F_{t}^{\prime}, t \in T^{\prime}$, induces a subsheaf $F_{t}^{\prime \prime}$ of $E^{\prime \prime}$. Now we copy the remaining part of the proof of Lemma 1.2 with trivial modifications. If for some integer $i$ the fibers $F_{t}^{\prime \prime} \mid\left\{Q_{i}\right\}, t \in T^{\prime}$, at $Q_{i}$ of these subsheaves are not constant, then in general we will not obtain a flat family of subsheaves of $E^{\prime \prime}$ and the integer $\operatorname{deg}\left(F_{t}^{\prime \prime}\right)$ may depend on $t$. Assume that for every $Q_{i}$ these fibers are not constant. Then for at least one $t \in T^{\prime}$ we have $F_{t}^{\prime} \mid\left\{Q_{i}\right\} \cap V_{i}^{\prime} \neq\{0\}$ for every $i$. Fix any such $t$. We have $\operatorname{deg}\left(F_{t}^{\prime \prime}\right) \leq F_{t}^{\prime}-u=\operatorname{deg}\left(F_{t}\right)-k$ and hence $s_{k}(E)=s_{k}(E(-Q)) \leq k(\operatorname{deg}(E(-Q)))-r\left(\operatorname{deg}\left(F_{t}^{\prime \prime}\right)\right) \leq k(\operatorname{deg}(E))-r\left(\operatorname{deg}\left(F_{t}\right)\right)-2 u$. Now we assume that the family of fibers $\left\{F_{t}^{\prime} \mid\{Q\}\right\}_{t \in T}$ is constant. We repeat the construction finitely many times until the corresponding family of fibers over $Q$ is not constant and we conclude as in the proof of Lemma 1.2.

Remark 1.5. The proofs of [9], Prop. 2.1 and Cor. 2.2, work verbatim when $X$ is a singular projective curve with arithmetic genus $g \geq 2$. Hence every vector bundle on $X$ is the flat limit of a flat family of stable vector bundles on $X$.

Remark 1.6. Let $E$ be a rank $r$ torsion free sheaf. Fix an integer $k$ with $0<$ $k<r$ and let $A$ be a rank $k$ saturated subsheaf of $E$ with $\operatorname{deg}(A)$ maximal, i.e. computing $s_{k}(E)$. Set $d:=\operatorname{deg}(E)$. The slope of a torsion free sheaf $B$ is a rational number with $\operatorname{rank}(B)$ as denominators. Hence we have some restrictions on the denominators of $\mu(E)-\mu(A)$ and hence on $s_{k}(E)$. Assume that we may apply 0.1 to $E$, i.e. assume that $X$ has only smoothable singularities and that $E$ is a flat limit of vector bundles. Then the inequality $s_{k}(E) \leq \operatorname{gk}(r-k)$ given by 0.1 may be refined in the following way (see [9], Th. 4.4, or [10], Cor. 3.13 and Remark 3.14). Let $\varepsilon$ be the only integer with $0 \leq \varepsilon \leq r-1$ and $\varepsilon+k(r-k)(g-1) \equiv k d$ modulo $r$. Then $s_{k}(E) \leq \varepsilon+k(r-k)(g-1)$.

Proof of Theorem 0.1. By assumption there is a flat family of torsion free sheaves $\left\{E_{t}\right\}_{t \in T}$ with $T$ smooth integral affine curve and $0 \in E$ such that $E_{0} \cong E$ and $E_{t}$ is locally free for $t \in(T \backslash\{0\})$. By the properness of the subscheme of the relative Quot-scheme of parametrizing sheaves with fixed degree and rank, we have the lower semicontinuity of the Lange invariants, i.e. we have $s_{k}(E) \leq s_{k}\left(E_{t}\right)$ for general $t \in T$. Hence it is sufficient to prove the statement of 0.1 for a locally free sheaf. By Remark 1.5 every locally free sheaf is the flat limit of a family of stable vector bundles. Hence the proof just given shows that it is sufficient to prove 0.1 when $E$ is a stable vector bundle. Let $V(r, d)$ be the moduli scheme of 
stable vector bundles on $X$ with degree $d$ and rank $r$. It is known that $V(r, d)$ is a smooth irreducible variety of dimension $r^{2}(g-1)+1$ ([16], Remark at p. 167). By the proof just given it is sufficient to check that for every integer $d$ there is a non-empty open subset $V^{\prime}(r, d)$ such that 0.1 is true for every $E \in V^{\prime}(r, d)$. We fix the integer $d$. For every $Q \in X$ let $c(Q)$ be the minimal positive integer length $\left(\boldsymbol{O}_{Q} / D_{Q}\right)$ such that there is an effective Cartier divisor $D$ with $D_{\text {red }}=\{P\}$. Hence $c(Q)=1$ if $Q \notin \operatorname{Sing}(X)$. Set $c(X):=\sup _{Q \in X} c(Q)$. Fix an integer $b$ with $b /(r-k)>d / r+2 g-2+c(X)$. For all stable vector bundles $A, B$ on $X$ with $\mu(A)<\mu(B)+2 g-2$ we have $h^{1}(X, \operatorname{Hom}(A, B))=h^{0}\left(X, \operatorname{Hom}\left(B, A \otimes \omega_{X}\right)\right)=0$, because the sheaf $A \otimes \omega_{X} \cong \operatorname{Hom}\left(A^{*}, \omega_{X}\right)$ is stable with slope $-\mu(A)+2-2 g$ even if $\omega_{X}$ is not locally free (see e.g. [2], Remark 1.4, or the discussion here at the beginning of section 2). Hence by the definition of the integer $c(X)$ it is straightforward to check that for every $A \in V(r, d)$ and every $B \in V(r-k, b)$ the vector bundle $\operatorname{Hom}(A, B)$ is spanned by its global section, $h^{1}(X, \operatorname{Hom}(A, B))=0, h^{0}(X, \operatorname{Hom}(A, B))=$ $(r-k) d-r b-r(r-k)(g-1)$. The fact that $\operatorname{Hom}(A, B)$ is spanned by its global sections implies that a general $f \in H^{0}(X, \operatorname{Hom}(A, B))$ is surjective. Thus for a general $f \in H^{0}(X, \operatorname{Hom}(A, B))$ the sheaf $\operatorname{Ker}(f)$ is a rank $k$ vector bundle with degree $d-b$. We claim that if $b$ is large, $A$ and $B$ are general and $f$ is general, then $\operatorname{Ker}(f)$ is stable. To check the claim we will reverse the construction. The dual construction shows that for large $b$ for every $A \in V(r, d)$, every $D \in V(k, d-b)$ and a general $f^{\prime} \in H^{0}(X, \operatorname{Hom}(D, A))$ the map $f^{\prime}$ is injective and with locally free cokernel, i.e. $\operatorname{Coker}\left(f^{\prime}\right)$ is a rank $r-k$ vector bundle with degree $b$. Furthermore, the simpleness of $A$ implies $h^{0}(X, \operatorname{Hom}(B, \operatorname{Ker}(f)))=h^{0}\left(X, \operatorname{Hom}\left(\operatorname{Coker}\left(f^{\prime}\right), D\right)\right)=0$, and hence by Riemann-Roch for all such $A, B, f, D$ and $f^{\prime}$ we have $\operatorname{dim}\left(\operatorname{Ext}^{1}(X, B, D)\right)=$ $\operatorname{dim}\left(\operatorname{Ext}^{1}\left(X, \operatorname{Coker}\left(f^{\prime}\right), D\right)\right)=\operatorname{dim}\left(\operatorname{Ext}^{1}(X, B, \operatorname{Ker}(f))\right)=b k-(d-b)(r-k)+$ $k(r-k)(g-1)$. Hence by the theory of the relative Ext-functor ([3] or [10]) and the openness of stability we obtain the claim, i.e. that for large $b$ a general $E \in V(r, d)$ fits in an exact sequence

$$
0 \rightarrow H \rightarrow E \rightarrow G \rightarrow 0
$$

with $H$ and $G$ stable vector bundles, $\operatorname{rank}(H)=k, \operatorname{rank}(G)=r-k$ and $\operatorname{deg}(G)$ large. We fix any such large integer $b$ and take as $V^{\prime}(r, d)$ the non-empty open subset of the stable bundles fitting in such exact sequence (1) with $H$ and $G$ stable. The Quot-scheme of $E$ at $G$ has dimension

$$
h^{0}(X, \operatorname{Hom}(X, G))=k(r-k)(\mu(G)-\mu(H)+g-1)
$$

and hence by Lemma 1.4 we obtain $s_{k}(E) \leq k(r-k) g$, as wanted.

If $X$ has only planar singularities, then every stable torsion free sheaf on $X$ is the flat limit of a family of stable vector bundles ([17]). Hence Theorem 0.1 has the following immediate corollary.

Corollary 1.7. Assume that $X$ has only planar singularities. For every rank $r$ stable torsion free sheaf $E$ on $X$ we have $s_{k}(E) \leq k(r-k) g$.

However, if $X$ has only smoothable singularities Theorem 0.1 has an easier proof which we want to give in detail because this particular case of 0.1 is sufficient to obtain Corollary 1.7.

Proof of Theorem 0.1 when $X$ has smoothable singularities. By assumption there is a flat family of torsion free sheaves $\left\{E_{t}\right\}_{t \in T}$ with $T$ smooth integral affine curve 
and $0 \in E$ such that $E_{0} \cong E$ and $E_{t}$ is locally free for $t \in(T \backslash\{0\})$. By the properness of the subscheme of the relative Quot-scheme of $\left\{E_{t}\right\}_{t \in T}$ parametrizing sheaves with fixed degree and rank, we have the semicontinuity of the Lange invariants, i.e. we have $s_{k}(E) \leq s_{k}\left(E_{t}\right)$ for general $t \in T$. Hence it is sufficient to prove the result for a locally free sheaf. Since $X$ has only smoothable singularities, $X$ is the flat limit of a flat family of smooth connected curves of genus $g$, i.e. there is a flat family of curves $\left\{X_{z}\right\}_{z \in Z}$ with $Z$ smooth affine curve and $a \in Z$ with $X_{a} \cong X$ and $X_{z}$ smooth and connected for every $z \in(Z \backslash\{a\})$. Passing to a ramified covering of a neighborhood of $a$ in $Z$ and using induction on $\operatorname{rank}(E)$ one obtains easily the existence of a rank $r$ vector bundle $E$ on the total space of $\left\{X_{z}\right\}_{z \in Z}$ with $E \mid X_{a} \cong E$ (up to the fixed isomorphism of $X_{a}$ and $X$ ). Again, by the properness of the connected components of the relative Quot-scheme of $E$ over $Z$, we have $s_{k}(E) \leq s_{k}\left(E \mid X_{z}\right)$ for general $z \in Z$. Since $X_{z}$ is smooth for general $z$, we have $s_{k}\left(E \mid X_{z}\right) \leq \operatorname{gk}(r-k)$ by [15], as wanted.

\section{Proof of 0.2 AND Other EXAMPles}

There is a good duality theory for finitely generated torsion free modules over a one-dimensional local Cohen-Macaulay ring $R$ if we use the functor $\operatorname{Hom}\left(-, \omega_{R}\right)$. In particular if $M$ is such module, the natural map $M \rightarrow \operatorname{Hom}\left(\operatorname{Hom}\left(M, \omega_{R}\right), \omega_{R}\right)$ is an isomorphism. However, if $R$ is not Gorenstein, i.e. if $\omega_{R}$ is not locally free, the functor $\operatorname{Hom}(-, R)$ has not this biduality property and indeed for any local onedimensional Cohen-Macaulay ring there is a finitely generated rank 1 torsion free module $M$ such that the natural inclusion $M \rightarrow M^{* *}:=\operatorname{Hom}(\operatorname{Hom}(M, R), R)$ is not surjective, i.e. such that $M$ is not reflexive. When $X$ is an integral projective curve local duality shows that the functor $\operatorname{Hom}\left(-, \omega_{X}\right)$ has good biduality properties in the category of coherent torsion free sheaves and good behaviour with respect to the computations of degrees and hence of slopes. In particular $E$ is stable (resp. semistable) if and only if $\operatorname{Hom}\left(E, \omega_{X}\right)$ is stable (resp. semistable). If $X$ is locally Gorenstein integral projective, then the same is true for the functor $\operatorname{Hom}\left(-, \boldsymbol{O}_{X}\right)$, i.e. a torsion free sheaf is stable (resp. semistable) if and only if its dual is stable (resp. semistable). This is false if $X$ is not locally Gorenstein (see Example 2.2). The aim of this section is to show the existence of several integral projective curves, $X$, on which torsion free sheaves may have pathological properties. In all examples there will be $P \in \operatorname{Sing}(X)$ such that $X$ is not Gorenstein at $P$. We recall the following easy fact (see e.g. [2], Remark 1.14).

Lemma 2.1. Let $X$ be an integral projective curve. Fix an integer $d, P \in X, a$ finitely generated torsion free module $M$ over the ring $\boldsymbol{O}_{X, P}$ and a finitely generated torsion free module $N$ over the completion $\boldsymbol{O}_{X, P}$. Then there exist stable torsion free sheaves $A$ and $B$ such that the localization of $A$ at $P$ is isomorphic to $M$ and the completion of the localization of $B$ at $P$ is isomorphic to $B$ and such that the restrictions of $A$ and $B$ to $Y \backslash\{P\}$ are locally free.

Example 2.2. Let $X$ be an integral projective curve such that there is $P \in$ $\operatorname{Sing}(X)$ whose local ring $R:=\boldsymbol{O}_{X, P}$ is not Gorenstein. Let $M$ be a finitely generated torsion free $R$-module which is not reflexive. Let $A$ be a stable torsion free sheaf on $X$ whose localization at $P$ is isomorphic to $M$. Take locally free sheaves $B$ and $D$ on $X$ with $\operatorname{rank}(B)=\operatorname{rank}(D)=\operatorname{rank}(A)$ and $\operatorname{deg}(B)=\operatorname{deg}(A)=\operatorname{deg}(D)-1$. Since $A^{* *}$ strictly contains $A$ we have $\operatorname{deg}\left(A^{* *}\right)>\operatorname{deg}(A)$. Set $E:=A \oplus B$. Obviously $E$ is semistable but $E^{* *}=A^{* *} \oplus B$ is not semistable. Now we consider torsion 
free sheaves which are extensions of $D$ by $A$, i.e. we consider exact sequences

$$
0 \rightarrow A \rightarrow F \rightarrow D \rightarrow 0 .
$$

By the local-to-global spectral sequence of the Ext-functors we have $\operatorname{Ext}^{1}(X ; D, A)$ $\cong H^{0}\left(X, \operatorname{Ext}^{1}(D, A)\right) \oplus H^{1}(X, \operatorname{Hom}(D, A))$. Since $A$ is locally free on $X \backslash\{P\}$, the sheaf $\operatorname{Ext}^{1}(D, A)$ is supported by $P$. Take any extension (2) whose image in $H^{0}\left(X, \operatorname{Ext}^{1}(D, A)\right)$ is zero, i.e. such that the corresponding extension of $R$-modules splits. Since $A$ and $D$ are locally free outside $P$ and $D$ is locally free, we obtain an exact sequence

$$
0 \rightarrow A^{* *} \rightarrow F^{* *} \rightarrow D \rightarrow 0 .
$$

Since $\operatorname{deg}\left(A^{* *}\right) \geq \operatorname{deg}(A)+1=\operatorname{deg}(D), F^{* *}$ is not stable. Now take any such $F$ for which (2) does not split. Since $h^{1}(X, \operatorname{Hom}(D, A))=h^{0}\left(X, \operatorname{Hom}\left(\operatorname{Hom}(D, A), \omega_{X}\right)\right)$ by Serre duality and $h^{0}\left(X, \operatorname{Hom}\left(\operatorname{Hom}(D, A), \omega_{X}\right)\right) \neq 0$ by Riemann-Roch, there exists such an extension. We want to check that $F$ is stable and hence that $F$ gives the example we were looking for. To simplify the arithmetic we will check the stability of $F$ when $\operatorname{deg}(A)=0$. In order to obtain a contradiction we assume the existence of a proper subsheaf $U$ of $F$ with $\mu(U) \geq \mu(F)=1 /(2 \operatorname{rank}(A))$. If $U$ is contained in $A$ we have $\mu(U) \leq \mu(A)=0$ by the semistability of $A$. Hence the image, $B$, of $U$ in $D$ is not zero. We have $B \cong U /(U \cap A)$. We distinguish three cases.

Case 1) Here we assume $U \cap A=\{0\}$. If $B=D, B$ induces a splitting of (2), contradicting the choice of $F$. If $B \neq D$, we have either $\operatorname{rank}(B)<\operatorname{rank}(D)$ or $B$ is not saturated in $D$. Since $\mu(D)=1 / \operatorname{rank}(D)$ and $D$ is stable, we obtain $\operatorname{deg}(B) \leq 0$. Hence $\operatorname{deg}(D) \leq 0$, contradiction.

Case 2) Here we assume $0<\operatorname{rank}(U \cap A)<\operatorname{rank}(A)$. Since $A$ is stable and $\operatorname{deg}(A)=0$, we have $\operatorname{deg}(U \cap A)<0$. Since $\operatorname{deg}(U)>0$, we have $\operatorname{deg}(B) \geq 2$. Since $\operatorname{rank}(B) \leq \operatorname{rank}(D)$, we obtain $\operatorname{deg}(B)>\operatorname{deg}(D)$, contradicting the semistability of $D$.

Case 3) Here we assume $U \cap A=A$. Since $B \neq F$, we have either $\operatorname{rank}(B)<$ $\operatorname{rank}(D)$ or $B$ is not saturated in $D$. Since $\mu(D)=1 / \operatorname{rank}(D)$ and $D$ is stable, we obtain $\operatorname{deg}(B) \leq 0$. Hence $\operatorname{deg}(D) \leq 0$, contradiction.

Proposition 2.3. Fix an integer $g \geq 5$. There exists an integral projective curve $X$ with $p_{a}(X)=g, \operatorname{card}(\operatorname{Sing}(X))=1, X$ analytically unibranch at its singular point, $P$, such that there exists a rank 2 torsion free sheaf $E$ on $X$ fitting in an exact sequence

$$
0 \rightarrow D \rightarrow E \rightarrow B \rightarrow 0
$$

with $B \in \operatorname{Pic}(X), D^{* *} \in \operatorname{Pic}(X), \operatorname{dim}_{K}\left(D^{* *} / D\right)=3, D$ locally free on $X \backslash\{P\}$, $\operatorname{deg}(B)=\operatorname{deg}\left(D^{* *}\right)+g-1$ and such that the extension (4) represents a smooth point of $\operatorname{Quot}(E)$ at which $\operatorname{Quot}(E)$ has dimension $\operatorname{deg}(B)-\operatorname{deg}\left(D^{* *}\right)+1-g=$ $\operatorname{deg}(B)-\operatorname{deg}(D)+4-g<\operatorname{deg}(B)-\operatorname{deg}\left(D^{* *}\right)+1-g ; X$ has embedding dimension 3 at $P$. Vice versa, given any integral projective curve $Z$ of genus $g$ and $Q \in \operatorname{Sing}(Z)$ with $\boldsymbol{O}_{X, P} \cong \boldsymbol{O}_{Z, Q}$ we may find an exact sequence (4) with the same properties (with respect to the pair $(Z, Q)$ instead of the pair $(X, P)$ ).

Proof. Fix a pair $(X, P)$ with $P \in \operatorname{Sing}(X)$; later, we will give explicitly $\boldsymbol{O}_{X, P}$. Take any rank 2 torsion free sheaf $E$ which fits in an exact sequence (44) (even the splitted one) with the following properties: $B \in \operatorname{Pic}(X), D^{* *} \in \operatorname{Pic}(X), D \neq D^{* *}$, 
$D$ locally free on $X \backslash\{P\}, \operatorname{deg}(B)=\operatorname{deg}\left(D^{* *}\right)+g-1, h^{1}\left(X, \operatorname{Hom}\left(D^{* *}, B\right)\right)=0$ (i.e. by Riemann-Roch $\left.h^{0}\left(X, \operatorname{Hom}\left(D^{* *}, B\right)\right)=\operatorname{deg}(B)-\operatorname{deg}\left(D^{* *}\right)+1-g\right)$. Set $e:=\operatorname{length}\left(D^{* *} / D\right)$. By assumption we have $e>0$; we will find an example with $e=3$; indeed it is easy to check that we must have $e \geq 2$ because the maximal ideal of a local one-dimensional Cohen-Macaulay ring is reflexive (see [5, lines 20-26 of page 430). Call e the point of $\operatorname{Quot}(E)$ corresponding to the exact sequence (4). We claim that $\operatorname{Quot}(E)$ is smooth at $\mathbf{e}$ of dimension $h^{0}\left(X, \operatorname{Hom}\left(D^{* *}, B\right)\right)=\operatorname{deg}(B)-\operatorname{deg}(D)-e+1-g$. The claim and the existence of the exact sequence (4) on $X$ would prove the proposition for the curve $X$. Since $B$ is locally free, we have $\operatorname{Ext}^{1}(B, A)=0$. The one-dimensionality of $X$ and a trivial spectral sequence give $\operatorname{Ext}^{1}(X ; B, A)=\operatorname{Ext}^{2}(X ; B, B)=0$. Since $B$ is locally free, (44) is locally split and hence there is no local obstruction to deforming $B$ as a quotient of $E$ (see 8], p. 374, or 7], exp. 221, $\S 5$ ). Hence $\operatorname{Quot}(E)$ is smooth at e and of dimension $h^{0}(X, \operatorname{Hom}(D, B))(\underline{8}$, p. 374, or [7], exp. 221, $\S 5)$. Since $B$ is locally free, we have $h^{0}(X, \operatorname{Hom}(D, B))=h^{0}\left(X, \operatorname{Hom}\left(D^{* *}, B\right)\right)$, proving the claim. Notice that for two general line bundles $B, A$ on $X$ with $\operatorname{deg}(B) \geq \operatorname{deg}(A)+g-1$ we have $h^{1}(X, \operatorname{Hom}(A, B))=0$. Furthermore, given an ideal $I$ of $\boldsymbol{O}_{X, P}$ with $I^{* *}$ principal (i.e. defining a line bundle near $P$ ) and any $A \in \operatorname{Pic}(X)$ we may find a rank 1 torsion free sheaf $D$ on $X$ with $D$ locally free on $X \backslash\{P\}$ and $D^{* *} \cong A$. Hence for the existence of (4) it is sufficient to find a pair $(X, P)$ such that there exists an ideal $I$ with $I^{* *}$ principal and with $\operatorname{dim}_{K}\left(I^{* *} / I\right)=3$. We will work on the formal completion, but everything will be algebraizable and the explicit construction could even give a monomial space curve with that type of singularity. Since we want $X$ analytically unibranch at $P, \mathbf{K}[[t]]$ must be the normalization of $\boldsymbol{O}_{X, P}$. Set $\boldsymbol{O}_{X, P}:=\mathbf{K}\left[\left[t^{3}, t^{7}, t^{11}\right]\right]$. We will use the elementary correspondence between numerical semigroups and ideals of semigroup rings like $\mathbf{K}\left[\left[t^{3}, t^{7}, t^{11}\right]\right]$ (see [4] or [5] for the background). $\mathbf{K}\left[\left[t^{3}, t^{7}, t^{11}\right]\right]$ corresponds to the numerical semigroup $S:=\langle 3,7,11\rangle$, i.e. the subset of $\mathbf{N}$ given by $\{0,3,6,7, \rightarrow)$, where " $*, \rightarrow$ " means "all integers $>*$ ". Take as ideal the ideal corresponding to the numerical ideal $J:=(6,15, \rightarrow)$ which is contained in the principal numerical ideal $T:=(6)+S=(6,9,12,13,15, \rightarrow)$. We have $\operatorname{card}(T \backslash J)=3$. It is sufficient to check that $S:(S: J)=T$. Since any principal ideal is reflexive, it is sufficient to check that $S: J=T^{*}=(-6)+S=(-6,-3,0,1,3, \rightarrow)$. This is a straightforward computation.

Remark 2.4. Many integers different from 3 may be obtained for the value $\operatorname{dim}_{\mathbf{K}}\left(D^{* *} / D\right)$ for different ideals and different local rings playing with numerical semigroups as in the proof just given. For an example needed for the proof of Theorem [0.2, see Example 2.5

Example 2.5. Set $\boldsymbol{O}_{X, P}:=\mathbf{K}\left[\left[t^{3}, t^{13}, t^{17}\right]\right]$, i.e. consider the semigroup ring associated to the numerical semigroup $S:=\langle 3,13,17\rangle=\{0,3,6,9,12,13,15, \rightarrow\} \subset \mathbf{N}$. Since $\operatorname{dim}_{\mathbf{K}}\left(\mathbf{K}[[t]] / \mathbf{K}\left[\left[t^{3}, t^{13}, t^{17}\right]\right]\right)=9$, this may be realized only on curves of arithmetic genus at least 9 . $X$ has embedding dimension 3 at $P$. We consider the ideal of $\mathbf{K}\left[\left[t^{3}, t^{13}, t^{17}\right]\right]$ associated to the numerical ideal $J:=(9,15,18,19,21, \rightarrow)$ of $S$. Let $I$ be the ideal of $\mathbf{K}\left[\left[t^{3}, t^{13}, t^{17}\right]\right]$ associated to $J . J$ is contained in the principal ideal $T:=(6)+S=(6,9,12,15,18,19,21, \rightarrow)$ and we have $\operatorname{card}(T \backslash J)=2$. Since $S: J=T^{*}$, we obtain $T=J^{* *}$. Hence $I^{* *}$ is principal, i.e. free, and $\operatorname{dim}_{\mathbf{K}}\left(I^{* *} / I\right)=2$. 
M. Maruyama proved in [13, Prop. 3.14, that on a smooth curve of genus $g \geq 2$ every rank 2 vector bundle $E$ with $s_{1}(E)$ has infinitely many (indeed $\infty^{1}$ many) rank 1 subbundles with maximal degree (see e.g. the introduction of [12]). This is not true for singular curves.

Theorem 2.6. Fix an integer $g \geq 9$. There exists an integral projective curve $X$ with $g=p_{a}(X)$ and $P \in \operatorname{Sing}(X)$ such that there exists a rank 2 torsion free sheaf $G$ on $X, G$ locally free on $X \backslash\{P\}$ and such that $s_{1}(G)=g$ but $E$ has only finitely many rank 1 subsheaves computing $s_{1}(G) . X$ has embedding dimension 3 at $P$ and we may find one such curve $X$ with $X \backslash\{P\}$ smooth.

Proof. We divide the proof into two parts.

Part (a). Here we take any projective integral curve $X$ of genus $g \geq 3$ and any $P \in X$; we will need only the case $P \in \operatorname{Sing}(X)$. The aim of this part of the proof is the construction of a rank 2 vector bundle $G$ on $X$ with certain properties listed in the First Claim below and at the very end of this part. Let $G(X, d)$ be the set of all rank 1 torsion free sheaves, $L$, on $X$ with $\operatorname{deg}(L)=d, h^{0}(X, L) \geq 2$ and $L$ spanned by $H^{0}(X, L)$. For all $A, M \in \operatorname{Pic}(X)$ with $\operatorname{deg}(A)=\operatorname{deg}(M)$, let $S(X ; A, M ; d)$ be the set of all rank 1 saturated subsheaves, $L$, of $A \oplus B$ with $\operatorname{deg}(L)=\operatorname{deg}(A)-d$. For any rank 2 vector bundle $F$ on $X$, let $S(F, t)$ be the set of all saturated rank 1 torsion free subsheaves of $F$ with degree $t$. Using Clifford's theorem proved in [6] it is easy to check that for every integer $d$ with $0<d \leq g-1$ we have $\operatorname{dim}(G(X, d)) \leq d-2$. Hence by semicontinuity we obtain that for general $A, M \in \operatorname{Pic}^{2}(X)$ and every integer $d$ with $0<d \leq g-2$ we have $\operatorname{dim}(S(X ; A, M, d)) \leq d-2$. We fix such general $A, M \in \operatorname{Pic}^{2}(X)$ and call $G$ the general vector bundle obtained from $A \oplus M$ making $g-2$ general positive elementary transformations supported by points of $X_{\text {reg }}$. Hence $\operatorname{deg}(G)=g$. Since $A$ and $M$ are subsheaves of $G$, we have $s_{1}(G) \geq g-2$. By the generality of the positive elementary transformations just made and the assumption $A \neq M$, the subsheaves $A$ and $M$ are saturated subsheaves of $G$.

First Claim: (i) $s_{1}(G)=g-2$;

(ii) $A$ and $M$ are the only subsheaves computing $s_{1}(G)$;

(iii) $S(G, 1)$ is finite or empty.

Notice that assertion (ii) of the claim implies that assertion (iii) of the claim is equivalent to the assertion that $G$ has only finitely many rank 1 subsheaves with degree 1 and not contained in the subsheaf $A$ or in the subsheaf $M$.

For every integer $d$ with $0 \leq d \leq g-2$ call $G(d)$ any bundle obtained from $A \oplus M$ making $d$ general positive elementary transformations. Hence $G(0)=A \oplus M$ and we may take $G=G(d)$.

Second Claim: For all integers $d, k$ with $0 \leq d \leq g-2$ and $d+1-g \leq k \leq 1$ we have:

(i) $s_{1}(G(d))=g-2$;

(ii) $A$ and $M$ are the only subsheaves computing $s_{1}(G(d))$;

(iii) $\operatorname{dim}(S(G(d), k)) \leq 1-k$.

Notice that for $d=g-2$ and $k=1$ the Second Claim gives the First Claim.

Proof of the Second Claim. We will check by induction on $d$ the three assertions of the Second Claim for all integers $k$. For $d=0$ these assertions are true because $\operatorname{dim}(S(X ; A, M ; k+2)) \leq k$ for every integer $k$. Assume the Second Claim for some integer $d-1$ with $1 \leq d \leq g-2$. Applying a general positive elementary transformation to $G(d-1)$ to obtain $G(d)$ we may assume that for all integers $k$ 
and all irreducible components, $T$, of $S(G(d-1), k)$ with $\operatorname{dim}(T)=1-k$ (if any) a general $L \in T$ remains saturated in $G(d)$. Hence we obtain the Second Claim.

By induction on $d$ we may even assume that for all integers $d$ with $0 \leq d \leq g-2$ every saturated rank 1 subsheaf $L$ of $G(d)$ with $\operatorname{deg}(L)=1$ has as fiber $L \mid\{P\}$ at $P$ a one-dimensional linear subspace of the two-dimensional vector space $G(d) \mid\{P\}$ which is different from the fiber $A \mid\{P\} \cong \mathbf{K}$ of $A$ at $P$ and that the same is true for all except finitely many ones of the rank 1 saturated subsheaves of degree 0 .

Part (b). Now we assume that $X$ has a singular point $P$ such that $\boldsymbol{O}_{X, P}$ is isomorphic to the local complete domain constructed in Example2.5 and such that the corresponding ideal $I$ with $I^{* *}$ free and $\operatorname{dim}_{\mathbf{K}}\left(I^{* *} / I\right)=2$ is algebraizable. Take the vector bundle $F$ given by Part (a). Up to a twist by a line bundle we may assume that $F$ fits in an exact sequence

$$
0 \rightarrow A \rightarrow F \rightarrow B \rightarrow 0
$$

with $A \in \operatorname{Pic}(X), B \in \operatorname{Pic}(X), \operatorname{deg}(A)=2$ and $\operatorname{deg}(B)=g$. Take the completion $F_{P}$ and $B_{P}$ of $F$ and $B$ at $P$ and identify $F_{P}^{\widehat{P}}$ with $\boldsymbol{O}_{\widehat{X}, P} \oplus \boldsymbol{O}_{X, P}$ in such a way that the second factor goes isomorphically onto $B_{P}$. Then see $I$ as an ideal of the first factor with bidual isomorphic to that factor. Since $I$ is algebraizable, there is a subsheaf $E$ of $F$ with $F / E$ skyscraper sheaf supported by $P$, $\operatorname{deg}(F / E)=\operatorname{dim}_{\mathbf{K}}\left(\boldsymbol{O}_{X, P}^{\widehat{a}} / I\right)>0$ and with $E$ formally isomorphic to $\boldsymbol{O}_{X, P} \oplus I$ at $P$. By construction the exact sequence (5) induces a surjection $\pi: E \rightarrow B$. Set $D:=\operatorname{Ker}(\pi)$. Hence $D$ is torsion free rank 1 sheaf with $D^{* *} \cong A$ and we have an exact sequence

$$
0 \rightarrow D \rightarrow E \rightarrow B \rightarrow 0 .
$$

We have $\operatorname{deg}(E)=g$ and $G \cong E^{* *}$. By construction the extension (6) induces a trivial extension at $P$. We claim that $s_{1}(E)=g$ and that $E$ has only finitely many degree 0 rank 1 subsheaves. Since $\operatorname{deg}(D)=0$, we have $s_{1}(E) \leq g$. Take a rank 1 torsion free subsheaf $R$ of $E$ computing $s_{1}(E)$; if $\operatorname{deg}(R)=0$, assume $R \neq D$ (as subsheaf of $E$, not just non-isomorphic to $D$ as abstract sheaf). Since $F$ is locally free and $G \cong E^{* *}$, the inclusion $R \rightarrow E$ induces an inclusion $R^{* *} \rightarrow G$. Since $s_{1}(G)=g-2$, we have $\operatorname{deg}\left(R^{* *}\right) \leq 2$. By part (ii) of the First Claim and the last property of $G$ given in Part (a) none of the saturated subsheaves of $G$ with degree 2 or 1 are subsheaves of $E$, while this may be true only for finitely many degree 0 saturated subsheaves.

Proof of Theorem 0.2. Fix a triple $(X, P, G)$ satisfying the thesis of Theorem 2.6 Fix $Q \in X_{\text {reg }}$ and let $E$ be the general torsion free sheaf obtained from $G$ making a negative elementary transformation supported by $Q$. Hence $E$ is a subsheaf of $G$ and $G / E$ is the skyscraper sheaf of length 1 with $Q$ as support. Let $L$ be a rank 1 saturated subsheaf of $E$ computing $s_{1}(E)$. Let $L^{\prime}$ be the saturation of $L$ in $G$. Since $s_{1}(G)=g$ we have $\operatorname{deg}\left(L^{\prime}\right) \leq \operatorname{deg}(G) / 2-g$ and $\operatorname{deg}\left(L^{\prime}\right)=\operatorname{deg}(G) / 2-g$ if and only if $L^{\prime}$ computes $s_{1}(G)$. If $L \neq L^{\prime}$, then $\operatorname{deg}(L) \leq \operatorname{deg}\left(L^{\prime}\right)-1 \leq \operatorname{deg}(G) / 2-g-1=$ $\operatorname{deg}(E) / 2-g-1 / 2$. Since the negative elementary transformation used to obtain $E$ from $G$ is general, and $G$ has only finitely many no such maximal subsheaf of $G$ is contained in $E$, i.e. if $L=L^{\prime}$, then $\operatorname{deg}\left(L^{\prime}\right) \leq \operatorname{deg}(G) / 2-g-1$, concluding the proof. 


\section{REFERENCES}

1. E. Ballico, On the number of components of the moduli schemes of stable torsion-free sheaves on integral curves, Proc. Amer. Math. Soc. 125 (1997), 2819-2824. MR 98d:14013

2. __ Stable sheaves on reduced projective curves, Ann. Mat. Pura Appl. (4) 175 (1998), 375-393. MR 2001a:14029

3. C. Banica, M. Putinar, and G. Schumaker, Variation deg globalen Ext in Deformationen kompakter Räume, Math. Ann. 250 (1980), 135-155. MR 82e:32015

4. V. Barucci, D. E. Dobbs, and M. Fontana, Maximality properties in numerical semigroups and applications to one-dimensional analytically irreducible local domains, Memoirs Amer. Math. Soc. 598, 1997. MR 97g:13039

5. V. Barucci and R. Fröberg, One-dimensional almost Gorenstein rings, J. Algebra 188 (1997), 418-442. MR 98a:13033

6. D. Eisenbud, J. Harris, J. Koh, and M. Stillman, Determinantal equations for curves of high degree, by D. Eisenbud, J. Koh, M. Stillman, Amer. J. Math. 110 (1988), 513-519. MR 89g:14023

7. A. Grothendieck, Fondements de la géométrie algébriques (extraits du Séminaire Bourbaki), Secrétarial Math., Paris, 1962. MR 26:3566

8. R. Hartshorne and A. Hirschowitz, Cohomology of a general instanton bundle, Ann. Sci. Ecole Norm. Sup. (4) 15 (1982), 365-390. MR 84c:14011

9. A. Hirschowitz, Problémes de Brill-Noether en rang supérieur, C. R. Acad. Sci. Paris Sér. I Math. 307 (1988), 153-156. MR 89i:14010

10. H. Lange, Universal families of extensions, J. Algebra 83 (1983), 101-112. MR 86e:14006

11. H. Lange, Zur Klassifikation von Regelmannigfaltigkeiten, Math. Ann. 262 (1983), 447-459. MR 85b:14019

12. H. Lange and M. S. Narasimhan, Maximal subbundles of rank two vector bundles, Math. Ann. 266 (1983), 55-72. MR 85f:14013

13. M. Maruyama, On the classification of ruled surfaces, Lecture in Mathematics Kyoto Univ. No. 3, Tokyo, 1970. MR 43:1990

14. M. Maruyama, Elementary transformations of algebraic vector bundles. In: Algebraic Geometry - Proceedings La Rabida, pp. 241-266, Lecture Notes in Math. 961, Springer-Verlag, 1981. MR 85b:14020

15. S. Mukai and F. Sakai, Maximal subbundles of vector bundles on a curve, Manuscripta Math. 52 (1985), 251-256. MR 86k:14013

16. P. E. Newstead, Introduction to moduli problems and orbit spaces, Tata Inst. Lecture Notes, Springer-Verlag, 1978. MR 81k:14002

17. C. J. Rego, Compactification of the space of vector bundles on a singular curve, Comm. Math. Helv. 57 (1982), 226-236. MR 84f:14015

Dipartimento di Matematicà, Università di Trento, 38050 Povo (TN) - Italy

E-mail address: ballico@science.unitn.it 